\title{
A survey of women's birth experiences in Scotland using the Birth Satisfaction Scale (BSS)
}

\author{
Caroline J. Hollins Martin BSc MPhil PhD PGCE ADM RM RGN MBPsS ${ }^{a}$ and Colin R. Martin \\ BSc MSc PhD MBA RN YCAP FHEA CPsychol CSci AFBPsS ${ }^{b}$
}

a Professor of Maternal Health, School of Nursing, Midwifery and Social Care, Edinburgh Napier University, Edinburgh, UK b Professor of Mental Health, Faculty of Society and Health, Buckinghamshire New University, Uxbridge Campus, Uxbridge, Middlesex, UK

\begin{abstract}
Background: 'Birth satisfaction' is important since experience of labour can influence women's post-natal adjustment.

Objective: To explore: (1) childbearing women's experiences of 'birth satisfaction' and (2) identify contexts in which it is more appropriate to use the 30-item Birth Satisfaction Scale (BSS) or the 10-item BSS-Revised (BSS-R).

Study design: A quantitative survey was conducted using the 30-item BSS. As a function of completing the 30-item BSS, participants also completed the short-form 10-item BSS-R.

Participants: A convenience sample of post-natal women $(n=228)$ attending for National Health Service (NHS) care at the Ayrshire Maternity Unit in the West of Scotland (UK) participated. Participants were within their first 10 post-natal days.

Results: The 30-item BSS mean scores $=115.84$ (SD 14.05). The thematically determined subscale mean scores of quality of care provision (BSS-QC; 8-items), women's personal attributes (BSS-WA; 8-items) and stress experienced during labour (BSS-SL;14-items) subscales, were 32.2 (4.09), 31.9 (4.16) and 51.73 (8.04) respectively. Women experienced greater 'birth satisfaction' when they had a Spontaneous Vertex Delivery compared with a complicated delivery. Primigravidas were generally less 'birth satisfied' than multiparous women.

Key conclusions: Overall assessment of care was complimentary, with global levels of ‘birth satisfaction’ generally high. In response to psychometric tests, the 30 -item BSS has been trimmed down to a statistically robust, valid and reliable 10 -item BSS-R.

Implications for practice: Now that a valid and reliable 10-item BSS has been developed to measure women's experiences of childbirth, it can be used to assess differences between variables such as home and hospital birth, or to establish correlates with other valid measures. We advance our study as an important contribution to person-centered maternal healthcare.
\end{abstract}

\section{Keywords}

Assessment, birth experience, birth satisfaction, Birth Satisfaction Scale (BSS), maternity care, midwives, obstetrics, person-centered healthcare, person-centered maternal healthcare

\section{Correspondence address}

Professor Colin R. Martin, Faculty of Society and Health, Buckinghamshire New University, Uxbridge Campus, 106 Oxford Road, Uxbridge, Middlesex, UB8 1NA, UK. E-mail: colin.martin@bucks.ac.uk

Accepted for publication: 6 May 2015

\section{Introduction}

The term 'birth satisfaction' represents survival of the woman and delivery of a healthy infant, with social policy documents in the UK advancing this definition to include provision of choice and control to childbearing women [14]. This paper assesses women's experiences of childbirth in just one large maternity unit in the West of Scotland (UK) using the Birth Satisfaction Scale (BSS) [5-7]. During data-analysis, items on the BSS were also validated and results are reported herein.

Within the literature many factors have been shown to influence women's satisfaction/dissatisfaction with their birth experience [5]. Factors include experiencing severe pain [8,9], excessive stress [8], being injured [10-13], receiving an obstetric intervention $[14,15]$, perceptions of inadequate medical care [16], long labour [17], personality characteristics $[18,19]$, being knowledgeable about birth $[20,21]$, self-efficacy [22,23], ability to cope with adversity [18], feeling in control [24-27], level of choice provided $[23,28,29]$, feeling empowered [20,30], labouring in a comfortable environment [31,32], continuous support throughout labour [24,33,34], quality relationships with care providers $[10,31,34]$ and delivery of a healthy infant [11,31].

Such evidence underpins factors that make a woman feel 'birth satisfied', which is important because negative 


\section{Table 1 Themes and subthemes of the Birth Satisfaction Scale (BSS) identified in a literature review (Hollins Martin \& Fleming, 2011) [5]}

\begin{tabular}{|ll|}
\hline (1) Quality of Care provision (QC) & (1a) Home assessment (Q12 \& 26) \\
& (1b) Birth environment (Q14 \& 28) \\
& (1c) Sufficient support (Q10 \& 14) \\
& (1d) Relationships with healthcare professional (Q13 \& 27) \\
\hline (2) Women's Attributes (WA) & (2a) Ability to cope during labour (Q1 \& 15) \\
& (2b) Feeling in control (Q12 \& 16) \\
& (2c) Preparation for childbirth (Q3 \& 17) \\
& (2d) Relationship with baby (Q11 \& 25) \\
\hline (3) Stress experienced during Labour (SL) & (3a) Distress experienced during labour (Q4 \& 18) \\
& (3b) Obstetric injuries (Q5 \& 19) \\
& (3c) Perception of having received sufficient medical care (Q7 \& 21) \\
& (3d) Receipt of an obstetric intervention (Q8 \& 22) \\
(3e) Pain experienced (Q29 \& 30) & (3f) Long labour (Q9 \& 23) \\
(3g) Health of baby (Q6 \& 20)
\end{tabular}

Participants responded to the 30-items on a 5-point Likert scale based on level of agreement/disagreement with the statement placed. Half of the items were reverse scored with post-natal scores ranging from 30-150. A score of 30 represents least satisfied and 150 most. An example question follows:

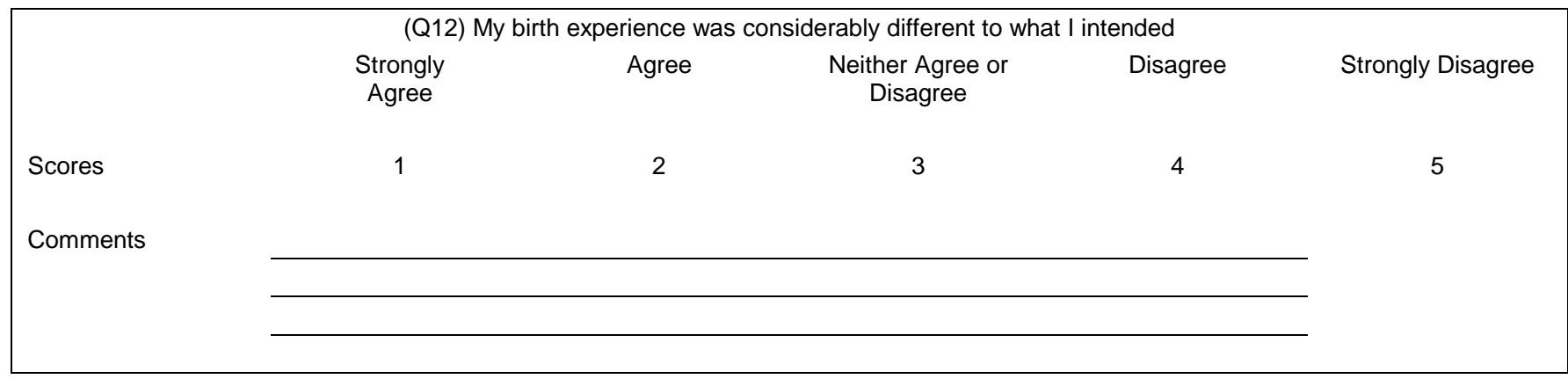

experiences of childbirth can be preserved across time $[10,35]$ and may impair mental wellbeing [36,37]. From the literature, Hollins Martin and Fleming [5] developed the 30-item Birth Satisfaction Scale (BSS) to improve professional understanding of what promotes birth satisfaction/dissatisfaction in one large maternity unit in the West of Scotland (UK). To view validation papers see Hollins Martin et al. [6] and Hollins Martin and Martin [7]. The research questions in this study asked: (1) What are childbearing women's experiences of birth satisfaction at the Ayrshire Maternity Unit in the West of Scotland (UK) and (2) How valid are the questions on the 30-item Birth Satisfaction Scale (BSS)?

\section{Method}

A quantitative survey of post-natal women was carried out between October 2010 and January 2011. Ethics approval was obtained from the UK National Health Service (NHS) National Research Ethics Service (NRES) (study reference: 10/S1001/31).

\section{Design}

The 30-item BSS [5] was validated [6-7] and used to assess post-natal women's levels of birth satisfaction. A short 10-item version of the BSS, the BSS-Revised (BSS$\mathrm{R}$ ), is intrinsic to the full scale and was developed by Hollins Martin and Martin [7] using structural equation modeling. The attendant community midwife collected data within the first 10 post-natal days.

\section{Participants}

A convenience sample of informed consenting post-natal women $(n=228)$ attending for National Health Service (NHS) care at the Ayrshire Maternity Unit (AMU) in the West of Scotland (UK) participated. Participants were recruited by the community midwives who delivered their post-natal care. The AMU has around 4,500 births a year and includes both a midwife led 'birthing unit' and an obstetric led 'delivery suite'. Participants were within their first 10 post-natal days. Inclusion criteria included uncomplicated pregnancy and term delivery between 37-43 weeks. The participant age range was 16-50 years. 
Exclusion criteria were delivery outwith term, medical diagnosis, suboptimal obstetric history or adverse obstetric clinical outcomes. Those younger than 16 and older than 50 years were excluded from the study. The population sampled were regular primigravid and multigravid women from the general community attending for routine UK National Health Service (NHS) care. NHS maternity service provision across the UK is standardised and regulated by the government, with women in the UK rarely seeking privatised care.

\section{Measuring tools}

The BSS is an instrument designed to measure women's levels of birth satisfaction on 15 domains underpinned by 3 themes (see Table 1).

To view items comprising the 30 -item BSS (see Table 2).

\section{Table 2 Birth Satisfaction Scale (BSS) (Hollins Martin \& Fleming, 2011) [5]}
(1) I coped well during my birth.
(2) The delivery room staff encouraged me to make decisions about how I wanted my birth to progress.
(3) I was well prepared for my labour, i.e., read a lot of literature and/or attended parenthood education classes.
(4) I found giving birth a distressing experience.
(5) I came through childbirth virtually unscathed.
(6) I gave birth to a healthy normal baby.
(7) During labour I received outstanding medical care.
(8) I received a lot of medical intervention, i.e., induction, forceps, section etc.
(9) I had a swift and speedy labour.
(10) I felt well supported by my partner during labour and birth.
(11) I was encouraged to hold my baby for a substantial amount of time after birth.

(12) My birth experience was considerably different to what I intended.

(13) I had the same midwife throughout the entire process of labour and delivery.

(14) I felt that the delivery room was unthreatening and comfortable.

(15) I felt very anxious during my labour and birth.

(16) I felt out of control during my birth experience.

(17) I felt it was better not to know in advance about the processes of giving birth.

(18) I was not distressed at all during labour.

(19) I felt mutilated by my birth experience.

(20) My baby was avoidably hurt during birth.

(21) The staff provided me with insufficient medical care during my birth.

(22) I had a natural labour, i.e., minimal medical intervention.

(23) I thought my labour was excessively long.

(24) I felt well supported by staff during my labour and birth.

(25) I was separated from my baby for a considerable period of time after my birth.

(26) My birth proceeded as I planned it.

(27) The staff communicated well with me during labour.

(28) The delivery room was clean and hygienic.

(29) Giving birth was incredibly painful.

(30) Labour was not as painful as I imagined.
The BSS was issued to post-natal women in their home by the community midwife on day 2 and collected before the health visitor took over care on day 10. A participant information sheet was issued, questions answered and a consent form signed.

\section{Data processing and analysis}

Data was entered into a software package (SPSS: PAWS statistics18) and statistical analysis conducted. A normal delivery was defined as a Spontaneous Vertex Delivery (SVD) and other as forceps, ventouse extraction, preorganised/emergency caesarean section and breech. Comparisons between groups were conducted using either the independent t-test or one-way analysis of variance (ANOVA). Associations between variables were assessed using Pearson's $\mathrm{r}$ correlation coefficient. Internal consistency was assessed using Cronbach's alpha.

\section{Results}

\section{Descriptive results}

A total of $(n=228)$ women completed the 30-item BSS and by derivation its associated subscales. As a function of completing the 30-item BSS, participants also completed the short-form 10 item BSS-Revised (BSS-R), which consists of the same subscales with reduced numbers of items (see Table 3).

Generally birth satisfaction scores were positive with lots of comments supporting that the participating women were satisfied with the maternity care they had received. Satisfaction was higher for women with Spontaneous Vertex Deliveries (SVD) compared with caesareans or other types of deliveries. In addition, satisfaction was higher for multiparous women in comparison to primiparous women. Satisfaction was also higher for women who had delivered in birthing units compared with theatre environments. Results for the two stated research questions are as follows:

\section{(Question 1) Women's levels of birth satisfaction}

One-hundred and ten (48\%) of the participants were primigravidas. The average duration of pregnancy was 40 (SD 1.26) weeks. The mean duration of labour was 8.6 (SD 6.81) hours. The total 30-item BSS score was 115.84 (SD 14.05) and the thematically determined subscale mean scores of the quality of care provision (BSS-QC; 8-items), women's personal attributes (BSS-WA; 8-items) and stress experienced during labour (BSS-SL; 14-items) subscales, were 32.20 (SD 4.09), 31.90 (SD 4.16) and 51.73 (SD 8.04) respectively. The short-form 10-item BSS-R score was 28.36 (SD 5.77) and the mean scores of the quality of care provision (BSS-R-QC;4-items), women's personal attributes (BSS-R-WA;2-items) and stress experienced during labour (BSS-R-SL; 4-items) 
Table 3 The 10 BSS-R items and associated domains derived by structural equation modeling (SEM) (Hollins Martin and Martin, 2014) [7]. Note: The SEM-derivation optimum model fit reported by Hollins Martin and Martin (2014) [7] produces a 4,4,2 item scale in contrast to their original thematicallyderived 4,3,3 item scale

\begin{tabular}{llll}
\hline Item & & Question & Domain \\
\hline 1 & $(5)$ & I came through childbirth virtually unscathed & Stress \\
2 & $(23)$ & I thought my labour was excessively long & The delivery room staff encouraged ne to make decisions about how I wanted my birth to \\
3 & $(2)$ & Quality & \\
& & progress & Attributes \\
4 & $(15)$ & I felt very anxious during my labour and birth & Quality \\
5 & $(24)$ & I felt well supported by staff during my labour and birth & Stress \\
6 & $(27)$ & The staff communicated well with me during labour & Attributes \\
8 & $(4)$ & I found giving birth a distressing experience & Stress \\
9 & $(16)$ & I felt out of control during my birth experience & \\
10 & $(18)$ & I was not distressed at all during labour & \\
\hline
\end{tabular}

Note: Original item number from the 30-item BSS in parentheses.

sub-scales, were 13.76 (SD 2.13), 4.9 (SD 1.98) and 9.7 (SD 3.29) respectively. To view the frequency table of birth characteristics by parity (see Table 4).

\section{Discriminant validity}

The mean 30-item BSS total score and thematicallyderived BSS-SL, BSS-WA and BSS-QC subscale scores and the 10-item BSS-R and structural equation model (SEM) derived BSS-R-SL, BSS-R-WA and BSS-R-QC subscale scores as a function of delivery type are shown in Table 5. A significant difference between groups differentiated by the delivery type (Spontaneous Vertex Delivery (SVD) or other (forceps, ventouse extraction, preorganised/emergency caesarian section or breech) was observed on BSS total score, $t_{(221)}=6.50, p<0.01$ and BSS-SL sub-scale score, $t_{(221)}=7.30, p<0.01$ in the direction predicted. Further and, against prediction, statistically significant differences were observed in BSSWA sub-scale scores, $t_{(221)}=3.22, p<0.01$, and BSS-QC sub-scale scores, $t_{(221)}=4.49, p<0.01$, as a function of type of childbirth. Significant difference between groups differentiated by delivery type have been reported elsewhere [7] using this dataset for the BSS-R total score and the BSS-R-SL sub-scale score (see Table 5 for mean scores and SD's).

The mean 30-item BSS total score and thematicallyderived BSS-SL (Stress experienced during Labour), BSSWA and BSS-QC subscale scores and the 10-item BSS-R and structural equation model (SEM) derived BSS-R-SL, BSS-R-WA and BSS-R-QC subscale scores as a function of parity are also shown in Table 5 .

Significant differences between groups differentiated by parity was observed on BSS total score, $t_{(226)}=3.61, p$ $<0.01$, BSS-SL subscale score, $t_{(226)}=3.19, p<0.01$ and BSS-QC subscale scores, $t_{(226)}=5.44, p<0.01$, with multiparous women having higher birth satisfaction scores compared to primiparous women.
Table 4 Frequency table of birth characteristics by parity

\begin{tabular}{lcc}
\hline & $\begin{array}{c}\text { Primigravida } \\
(n=110)\end{array}$ & $\begin{array}{c}\text { Multigra } \\
(n=118)\end{array}$ \\
\hline Birthing environment & & \\
Delivery suit & 68 & 82 \\
Birthing unit & 11 & 14 \\
Home & 0 & 2 \\
Theatre & 30 & 20 \\
Unknown & 1 & 0 \\
& & \\
Type of childbirth & & \\
& & 91 \\
SVD & 51 & 6 \\
Forceps & 24 & 1 \\
Ventouse & 12 & 12 \\
Pre-organised CS* & 1 & 4 \\
Emergency CS & 21 & 0 \\
Breech & 0 & 4 \\
Pool & 1 &
\end{tabular}

Pain relief

$\begin{array}{lcc}\text { Epidural } & 65 & 35 \\ \text { Opiates } & 15 & 13 \\ \text { Nitrous oxide } & 25 & 63 \\ \text { Alternative methods } & 2 & 1 \\ \text { None } & 2 & 6 \\ \text { Unknown } & 2 & 0\end{array}$

${ }^{*}$ Caesarian Section ${ }^{* *}$ e.g., reflexology, acupuncture, homeopathy SVD = Spontaneous Vertex Delivery. Note: a birthing unit or centre is a small maternity unit staffed and led by midwives with a remit of normal physiological birth. 
Table 5 Mean 30-item BSS and BSS thematically-determined subscales and 10-item BSS-R and BSS-R subscale scores categorised by delivery type, parity and delivery environment. Standard deviations in parentheses

\begin{tabular}{|l|c|c|c|c|c|c|c|}
\hline Variable & $\begin{array}{c}\text { SVD } \\
(\mathrm{n}=142)\end{array}$ & $\begin{array}{c}\text { Other } \\
(\mathrm{n}=86)\end{array}$ & $\begin{array}{c}\text { Primiparous } \\
(\mathrm{n}=110)\end{array}$ & $\begin{array}{c}\text { Multiparious } \\
(\mathrm{n}=118)\end{array}$ & $\begin{array}{c}\text { Delivery suite } \\
(\mathrm{n}=150)\end{array}$ & $\begin{array}{c}\text { Birthing unit } \\
(\mathrm{n}=25)\end{array}$ & $\begin{array}{c}\text { Theatre } \\
(\mathrm{n}=50\end{array}$ \\
\hline & & & & & & & \\
\hline BSS-total & $119.73(13.1)$ & $108.1(12.4)$ & $112.44(14.1)$ & $119(13.3)$ & $117(13.2)$ & $122.68(11.8)$ & $106.24(13.4)$ \\
\hline BSS-SL & $54.26(7.2)$ & $46.89(7.3)$ & $50.01(8.5)$ & $53.34(7.3)$ & $52.94(7.5)$ & $54.92(6.5)$ & $46.22(8.1)$ \\
\hline BSS-WA & $32.44(4.1)$ & $30.63(4)$ & $31.67(4.2)$ & $32.12(4.2)$ & $32.24(4)$ & $34.04(3.5)$ & $29.62(4.1)$ \\
\hline BSS-QC & $33.03(4)$ & $30.58(3.7)$ & $30.76(3.9)$ & $33.54(3.8)$ & $32.46(4.1)$ & $33.72(4)$ & $30.4(3.6)$ \\
\hline & & & & & & & \\
\hline BSS-R total & $29.19(5.9)$ & $26.5(5.1)$ & $27.68(5.8)$ & $28.99(5.7)$ & $28.92(5.7)$ & $30.08(6.2)$ & $25.58(5.2)$ \\
\hline BSS-R-SL & $10.39(3.2)$ & $8.3(3.1)$ & $9.08(3.3)$ & $10.27(3.2)$ & $10.13(3.1)$ & $10.52(3.5)$ & $7.94(3.3)$ \\
\hline BSS-R-WA & $5.11(2)$ & $4.46(1.8)$ & $4.83(2)$ & $4.97(2)$ & $5.05(2)$ & $5.52(1.6)$ & $4.08(2)$ \\
\hline BSS-R.QC & $13.68(2.2)$ & $13.75(2)$ & $13.77(2.1)$ & $13.75(2.1)$ & $13.75(2.2)$ & $14.04(2.4)$ & $13.56(1.9)$ \\
\hline & & & & & & & \\
\hline
\end{tabular}

SVD = Spontaneous Vertex Delivery; Other delivery = forceps, ventouse extraction, pre organised/emergency caesarian section or breech Home birth was removed from the analysis since only two women had this form of birth experience. Five women who had a pool birth were excluded from inferential statistical analysis in relation to delivery type due to low $\mathrm{N}$ and this not being a typical and universally available birthing experience in the UK.

There was no statistically significant difference between groups on BSS-WA subscale scores, $t_{(226)}=0.81$, $p=0.42$. Contrasting with these observations, significant differences between groups differentiated by parity were observed only on the BSS-R-SL subscale score, $t_{(226)}=$ 2.77, $p<0.01$, with multiparous women having greater birth satisfaction scores. In contrast, no significant differences as a function of parity were observed on BSS-R total score, $t_{(226)}=1.72, p=0.09$, BSS-R-WA subscale score, $t_{(226)}=0.56, p=0.58$ and BSS-R-QC sub-scale scores, $t_{(226)}=0.1, p=0.92$.

The mean 30-item BSS total score and thematicallyderived BSS-SL, BSS-WA and BSS-QC subscale scores and the 10-item BSS-R and structural equation model (SEM) derived BSS-R-SL, BSS-R-WA and BSS-R-QC subscale scores as a function of delivery environment type are also shown in Table 5.

One-way ANOVA revealed significant differences between groups on all long-form measures of the 30-item BSS; BSS total score, $F_{(2,222)}=18.26, p<0.01$, BSS-SL subscale score, $F_{(2,222)}=17.54, p<0.01$, BSS-WA subscale score, $F_{(2,222)}=12.39, p<0.01$ and BSS-QC subscale scores, $F_{(2,222)}=7.30, p<0.01$. Post hoc comparisons conducted using the Bonferroni procedure to control for family-wise error rate when conducting multiple comparisons. It was found that though there were no significant differences across any of the long-form 30-item BSS measure scores between the delivery suite and the birthing unit, long form 30-item BSS measure scores were significantly higher for delivery suite and birthing unit environments compared to the theatre environment (delivery suite/birthing unit vs. theatre, all comparisons $p<0.01)$.

\section{(Question 2) Validation of the BSS}

The thematically determined subscales of the 30-item BSS include quality of care provision (8-items), women's personal attributes (8-items) and stress experienced during labour (14-items). Similarly, again using three subscales, the short-form BSS-R uses a reduced item set of 4, 2 and 4 items to measure the quality of care provision, women's personal attributes and stress experienced during labour domains respectively. One-way ANOVA revealed mostly consistent findings to those of the long form 30-item BSS, with the 10-item BSS-R and associated subscales with one important exception. The ANOVA findings revealed a significant impact of childbirth environment on BSS-R total score, $F_{(2,222)}=8.05, p<0.01$, BSS-R-SL subscale score, $F_{(2,222)}=9.85, p<0.01$ and BSS-R-WA sub-scale score, $F_{(2,222)}=6.14, p<0.01$. Post-hoc comparisons conducted using the Bonferroni procedure revealed that there were no significant differences across any of the short-form 10-item BSS-R measure scores between the delivery suite and the birthing unit. However all post-hoc comparisons revealed delivery suite and birthing unit environments scores to be significantly higher than the theatre environment (delivery suite/birthing unit vs. theatre, all comparisons $p<0.01$ ). However, in contrast to the long-form BSS-QC subscale, no effect of childbirth environment was observed on BSS-R-QC subscale scores, $F_{(2,222)}=0.42, p=0.65$, thus post hoc comparisons were not conducted on this subscale.

\section{Divergent validity}

No significant correlation was observed between the shortform 10-item BSS-R total score and mother's age, $r=$ $0.07, p=0.28$. Neither was there evidence of any significant relationships with the short-form subscale 
scores: between BSS-R-SL scores, BSS-R-WA scores, BSS-R-QC and mother's age, $r=0.07, p=0.27 ; r=0.01$, $p=0.96 ; r=0.08, p=0.21$; respectively. However, longform 30-item BSS scores revealed a number of statistically significant correlations with mothers age, BSS-R total score, BSS-SL scores, BSS-WA scores, BSS-QC, $r=0.15$, $p=0.02 ; r=0.13, p=0.05 ; r=0.06, p=0.4, r=0.19$, $p<0.01$; respectively. Correlations between BSS and BSS$\mathrm{R}$ full scale and subscale scores are shown in Table 6 .

\section{Internal consistency}

Calculated Cronbach's alpha of the long form 30-item BSS total scale and thematically-derived BSS-SL, BSS-WA, BSS-QC subscales were $0.87,0.78,0.67$ and 0.66 respectively. The Cronbach's alpha of the 10-item BSS-R total scale and SEM-derived BSS-R-SL, BSS-R-WA, BSSR-QC subscales were $0.79, \quad 0.71,0.64$ and 0.74 respectively, these short-form alpha's having originally been reported by Hollins Martin and Martin [7] in the BSS-R development paper.

\section{Discussion}

Overall assessment of care was complimentary, with global levels of 'birth satisfaction' generally high. It is recognized that women who have good outcomes from childbirth are likely to have higher BSS scores than those who have negative outcomes. The first interesting finding was that women who received caesarean section reported reduced birth satisfaction compared with those who had Spontaneous Vertex Delivery (SVD). The literature also supports that obstetric intervention is linked with reduced birth satisfaction [15], which includes caesarean section $[38,39]$. The following quote written in the comments section of the 30-item BSS illustrates a commonly articulated worry that surrounds operative delivery:

"Birth went OK but got a bit distressed because of emergency section. Was just worried about baby.” (P152)

Out of the total $(n=228)$ participants, $(n=25)$ experienced emergency and $(n=13)$ elective CS. These experiences differ between the two groups in terms of birth satisfaction, with the emergency cluster gaining lower BBS scores than the planned cluster and both performing lower in means scores in comparison to other delivery groups. This informs us that the act of both emergency and elective abdominal surgery present as less satisfying experiences compared with vaginal birth. A larger study that explores birth satisfaction between these two groups (emergency CS/elective CS) that is triangulated by a qualitative investigation could provide valuable data.

In the 30-item BSS, pain experienced by childbearing women was assessed through questions 29 and 30 (see Table 2), with both considered not robust enough for retention on the valid and reliable 10 -item BSS. Since pain is a factor that causes women to become distressed and feel out of control, it is considered to be evaluated within the 10-item BSS in items 8 and 9 (see Table 3). A qualitative comments section beneath these questions provides space for participants to elaborate their thoughts about what caused them to become distressed or lose control during labour. Qualitative perspectives obtained through interviews would also provide more information on how pain effects childbearing women's birth satisfaction in terms of distress experienced and level of feeling in control during labour.

Significant psychological morbidity can arise from certain types of childbirth and in particular emergency caesarean section [40]. Operative delivery has also been associated with higher weighted scores on the Edinburgh Postnatal Depression Scale [20], with 30-item BSS scores significantly improved for delivery suite and birthing unit compared to theatre. The following quote emphasises satisfaction gained from vaginal birth:

"This birth was a pleasant surprise after a lot of medical intervention with my first child.” (P42)

Higher levels of mental health problems post-operative delivery, raises the question of how midwives/obstetricians tackle prevention of psychological consequences from having a caesarean section. Interventions may include: (1) careful history taking to determine prior trauma experiences that enhance fear of repetition, (2) affording excellent pain control and (3) providing post-partum care that incorporates empathy and congruence for the woman's birth experience [41]. The National Institute of Clinical Excellence (NICE) guideline 132 Issued in November 2011 (UK) [42] advises that maternal request for caesarian section for reasons of anxiety must be supported postcounselling, with this competing controversial topic fit for debate. The following quote highlights how even a planned section can be an unpleasant experience:

"Having a planned section was not a pleasant experience and recovery afterwards has been slow. The care at the hospital during and afterwards was exceptional.” (P204)

The fact that women's attributes (ability to cope, feel in control, self-educate and develop quality relationships) made no difference in terms of type of childbirth is perhaps because these characteristics are rooted in personality, with development involving encoding of internal models, schemas and premise systems [43]. Some psychologists believe in a fixed theory, whilst others embrace a malleable (or incremental) concept, with qualities of personality developing through motivational efforts and knowledge acquisition [44]. Our finding that personal attributes do not alter women's experiences of birth satisfaction supports the 'fixed trait' theory of personality.

One explanation for reduced birth satisfaction in primigravidas compared with multiparous women may be due to an increased tendency towards developing complications. For example, primigravidas are more likely to experience pregnancy-induced hypertension and its associated intrauterine growth retardation [45], with such 
Table 6 Correlations between 30-item BSS scores, thematically-derived BSS subscale scores, 10item BSS-R total and associated subscale scores (all correlations statistically significant at $p<0.01$ )

\begin{tabular}{|c|c|c|c|c|c|c|c|c|}
\hline Scale & BSS total & BSS-SL & BSS-WA & BSS-QC & BSS-R total & BSS-R-SL & BSS-R-WA & BSS-R-QC \\
\hline BSS-total & & 0.94 & 0.8 & 0.78 & 0.9 & 0.81 & 0.68 & 0.56 \\
\hline BSS-SL & & & 0.63 & 0.61 & 0.82 & 0.86 & 0.55 & 0.338 \\
\hline BSS-WA & & & & 0.48 & 0.8 & 0.59 & 0.82 & 0.49 \\
\hline BSS-QC & & & & & 0.67 & 0.48 & 0.43 & 0.68 \\
\hline BSS-R-total & & & & & & 0.86 & 0.8 & 0.63 \\
\hline BSS-R-SL & & & & & & & 0.57 & 0.26 \\
\hline BSS-R-WA & & & & & & & & 0.35 \\
\hline BSS-R-QC & & & & & & & & \\
\hline
\end{tabular}

adverse outcomes part of the exclusion criteria. The sorts of complications considered are those that can arise during labour. For example, longer labour and its perils of maternal exhaustion, fetal distress, obstetric intervention and associated infant harm [46].

Increased anxiety about complications may be just one contributor to differential birth satisfaction scores between women. Also, multiparous women harbour prior expectations founded in experience, as the following quotes illustrate:

“Prepared through previous experience.” (P126)

"Third baby. I understood about labour and knew how painful it would be.” (P165)

“I already knew what to do.” (P175)

An important finding relates to the quality of care subscale. Overall assessment was complimentary, with the following quotes selected to illustrate this:

\footnotetext{
"This was my first baby and my experience at Ayrshire Maternity Unit was wonderful. It was a great comfort to know everything was in hand and I didn't need to worry about a thing. From my arrival to when I left EVERY member of staff was considerate, professional and extremely caring. I think Ayrshire Maternity Unit is a wonderful unit and Ayrshire is fortunate to have it. The unit itself is obviously state of the art and very new but it's the staff that made it what it is.” (P201)
}

"The doctors and midwives at Ayrshire Maternity Unit were very supportive during my stay there. They're doing a fantastic job.” (P196)
“Fantastic staff and facilities. Couldn't have wished for better care.” (P16)

In terms of statistical validity, the 10-item BSS-R has demonstrated reliability and validity, with the additional 20 items on the 30-item BSS demonstrating insufficient levels of statistical validity [7]. In spite of this, concurrent analysis has accounted for the total scale content and promotes the 30-item BSS (14) as an all-encompassing measure of birth satisfaction [6].

As such, both versions of the BSS may be considered fit for differing purpose. In relation to validating the 30item BSS, no significant difference as a function of parity was observed on the 10-item BSS-R total score, which supports that the 30-item BSS should be selected for use when an assessor wishes to discriminate between parity status. Pending larger participant numbers and further data collection, this claim may be further validated.

Also, no significant correlation was observed between the 10-item BSS-R total score and mother's age, or for any of the BSS-R subscales and mother's age. In contrast, the 30-item BSS scores reveal significant correlations between mother's age and total score, stress experienced during labour and quality of care. Hence, when using the long version 30-item BSS age-related adjust for scores is recommended.

The data do not support that the 30-item BSS is a valid and reliable instrument for measuring birth satisfaction. However, as a questionnaire, it may be useful for gathering qualitative data, since it is evidence-based [5-7], with items directing identification of a broad-spectrum of particulars that relate to birth satisfaction. The 30-item BSS may be of particular use when providing person-centered care, especially when 'birth dissatisfaction' is verbally expressed and counselling is being considered, or as a screening tool prior to in-depth qualitative work. 
In contrast, the 10-item BSS-R, which has been shown to be statistically robust [7], is more useful for employing in quantitative research; for example, establishing correlates with other psychometric measures. The BSS has become an International instrument and is currently being used in Greece, Turkey, Taiwan, Czechoslovakia, Ireland, United States of America and England to collect birth satisfaction data. Hence, evidence is accumulating which indicates that the 30-item BSS and the revised 10-item BSS-R are valuable methods of measuring birth satisfaction in a variety of contexts and cultures.

One strength of the study is that the scale provides evidence-based variables that 'do' or 'do not' affect birth satisfaction. Items on the BSS were identified and reported in a literature-based paper by Hollins Martin and Fleming [5] and were further qualitatively validated using a research method called concurrent analysis [6]. Another strength is that the data gathered have ecological validity, given that they were amassed from authentic post-natal women by maternity care experts. In contrast, results could be criticised for representing only one maternity unit in one western culture and that the sample size was not representative of the larger population. In response, more quantitative studies and data comparisons in a variety of settings and cultures are recommended. The BSS can be requested from the first author.

\section{Conclusion}

Both quantitative and qualitative findings from the BSS demonstrate that childbearing women in just one region of the West of Scotland (UK) perceive that they are receiving quality maternity care. Results indicate that having a government led system funded by tax payers works for the majority of women, noting that the majority utilize this service regardless of socioeconomic grouping. Although we have focused on just one maternity unit and population, these results can be used in future cross-cultural contrasts, validity comparisons and future meta-analysis. Now that a valid and reliable 10-item BSS has been developed to measure women's experiences of childbirth, it can be used to assess differences between stand alone systems of care, for example, home and hospital birth, or to establish correlates with other valid measures, for example, selfefficacy, anxiety, depression, locus of control or bonding. It would also be interesting to undertake a longitudinal assessment of birth satisfaction beyond the first few days post-birth, for example, at 6 weeks, 6 months and beyond to explore cross-cultural experience.

\section{Acknowledgements and Conflicts of Interest}

We thank Geraldine Butcher and the community midwifery staff who hosted this research study. Their efforts during the data collection are greatly appreciated. We also thank the numerous fatigued post-natal women $(n=228)$ who co-operated with our requests. We are also very grateful to the reviewers of this manuscript for their valuable insights and helpful suggestions. We declare no conflicts of interest.

\section{References}

[1] Department of Health (DoH). (2004). The National Service Framework for Children, Young People and Maternity Services. London: DoH.

[2] Department of Health (DH). (2006). Our health, our care, our say. London: DoH.

[3] Department of Health (DoH). (2007). Maternity matters: choice, access and the continuity of care in a safe service. London: DoH.

[4] Department of Health (DoH). (2007). Improving the quality and outcomes for maternity service users through effective commissioning. London: DoH.

[5] Hollins Martin, C.J. \& Fleming, V. (2011). The Birth Satisfaction Scale (BSS). International Journal of Health Care Quality Assurance. 24 (2) 124-135.

[6] Hollins Martin, C.J., Snowden, A. \& Martin, C.R. (2012). Concurrent analysis: Validation of the domains within the Birth Satisfaction Scale. Journal of Reproductive and Infant Psychology 30 (3) 247-260.

[7] Hollins-Martin, C.J. \& Martin, C. (2014). Development and psychometric properties of the Birth Satisfaction Scale-Revised (BSS-R). Midwifery 30 (6) 610-619.

[8] Quinne, L., Rutter, D.R. \& Gowan, S. (1993). Women's satisfaction with the quality of the birth experience: a prospective study of social and psychological predictors. Journal of Reproductive and Infant Psychology 11, 107-113.

[9] Melzack, R., Taenzer, P., Feldman, P. \& Kinch, R. (1984). Labor is still painful after prepared childbirth. Canadian Medical Journal 125, 357-363.

[10] Sorenson, D.S. \& Tschetter, L. (2010). Prevalence of negative birth perception, disaffirmation, perinatal trauma symptoms, and depression among postpartum women. Perspectives in Psychiatric Care 46 (1) 14-25.

[11] Geissbuehler, V. \& Eberhard, J. (2002). Fear of childbirth during pregnancy: a study of more than 8000 pregnant women. Journal of Psychosomatic Obstetrics \& Gynaecology 23, 229-235.

[12] Beck, C.T. (2009). Birth trauma and its sequelae. Journal of Trauma \& Dissociation 10 (2) 189-203.

[13] McKenzie-McHarg, K. (2004). Traumatic birth: understanding predictors, triggers, and counseling process is essential to treatment. Birth 31 (3) 219-221.

[14] Gamble, J. \& Creedy, D. (2005). Psychological trauma symptoms of operative birth. British Journal of Midwifery 13 (4) 218-224.

[15] Jacoby, A. (1987). Women's preferences for and satisfaction with current procedures in childbirth: findings from a national study. Midwifery 3, 117-124.

[16] Eriksson, C., Westman, M.D. \& Hamberg, M.D. (2006). Content of childbirth related fear in Swedish women and men: analysis of an open ended questionnaire. American College of Nurse-Midwives 51, 112-118. 
[17] Morgan, B.M., Bulpitt, C.J., Clifton, P. \& Lewis, P.J. (1982). Analgesia and satisfaction in childbirth (the Queen Charlotte's 1000 mother survey). Lancet 2 (8302) 808-811. [18] Lowe, N.K. (1991). Maternal confidence in coping with labour. Journal of Obstetric, Gynecologic and Neonatal Nursing 20 (6) 457-463.

[19] Stockman, A. \& Altmaier, E. (2001). Relation of selfefficacy to reported pain and pain medication usage during labour. Journal of Clinical Psychology in Medical Settings 8, 161-166.

[20] Brown, S. \& Lumley, J. (1994). Satisfaction with care in labour and birth: a survey of 790 Australian women. Birth 21 (1) 4-13.

[21] Dannenbring, D., Stevens, M.J. \& House, A.E. (1997). Predictors of childbirth pain and maternal satisfaction. Journal of Behavioural Medicine 20 (2) 127142.

[22] Bandura, A. (1982). Self efficacy mechanism in humans. American Psychologist 37 (2) 122-147.

[23] Handfield, B. \& Bell, R. (1996). Do childbirth preparation classes influence decision making about labour and postpartum issues? Birth 22 (3) 153-160.

[24] Ford, E. \& Ayers, S. (2009). Stressful events and support during birth: the effect on anxiety, mood and perceived control. Journal of Anxiety Disorders 23 (2) 260-268.

[25] Goodman, P., Mackay, M.C. \& Tavakoli, A.S. (2004). Factors related to childbirth Satisfaction, Journal of Advanced Nursing 46, 212-219.

[26] Green, J.M., Baston, H.A., Easton, S.C. \& McCormick, F. (2003). Greater expectations. The interrelationship between women's expectations and experience of decision-making, continuity, choice and control in labor, and psychological outcomes. Leeds: Mother and Infant Research Unit, University of Leeds. Leeds, UK.

[27] Knapp, L. (1996). Childbirth satisfaction: the effects of internality and perceived control. Journal of Perinatal Education 5, 7-16.

[28] Gibbens, J. \& Thomson, A. (2001). Women's expectations and experiences of childbirth. Midwifery 17, 302-313.

[29] Hall, S.M. \& Holloway, I.M. (1998). Staying in control: women's experiences of labour in water. Midwifery 14, 30-36.

[30] Halldorsdottir, S. \& Karlsdottir, S.I. (1996). Journeying through labour and delivery: perceptions of women who have given birth. Midwifery 12, 48-61.

[31] Proctor, S. (1998). What determines quality in maternity care: comparing the perceptions of childbearing women and midwives. Birth 25 (2) 85-93.

[32] Waldenstrom, U. \& Nilsson, C.A. (1993). Women's satisfaction with birth centre care: a Randomised controlled study. Birth 21 (1) 3-13.

[33] Hodnett, E.D., Gates, S., Hofmeyr, G.J., Sakala, C.

(2012). Continuous support for women during childbirth.

Cochrane Database of Systematic Reviews. Issue 10. Art.

No.: CD003766. DOI:

10.1002/14651858.CD003766.pub4.

[34] Chen, C., Wang, S. \& Chang, M. (2001). Women's perceptions of helpful and unhelpful nursing behaviours during labour: a study of Taiwan. Birth 28 (3) 180-185.
[35] Stadlmayr, W., Amsler, F., Lemola, S., Stein, S., Alt, M., Bürgin, D., Surbek, D. \& Bitzer, J. (2006). Memory of childbirth in the second year: the long term effect of a negative birth experience and its modulation by the perceived intranatal relationship with caregivers. Journal of Psychosomatic Obstetrics \& Gynecology 27 (4) 211224.

[36] Bedell, S.E., Graboys, T.B., Bedell, E. \& Lown, B. (2004). Words that harm, words that heal. Archives of Internal Medicine 164 (13) 1365-1368.

[37] Kirkpatrick, J.N., Nash, K. \& Duffy, T.P. (2005). Well rounded. Archives of Internal Medicine 165 (6) 613616.

[38] Ryding, E.L., Wijma, B., Wijma, K. \& Rydstrom, H. (1998). Fear of childbirth during pregnancy may increase the risk for emergency section. Acta Obstetrica et Gynecologia Scandinavica 77, 542-547.

[39] Wax, J., Cartin, A., Pinette, M. \& Blackstone, J. (2004). Patient choice caesarean: an evidence based view. Obstetrical \& Gynecological Survey 59, 601-616.

[40] Hannah, P., Adams, D., Lee, A. \& Glover, V. (1992). Links between early post-partum mood and post-natal depression. British Journal of Psychiatry 160, 777-780.

[41] Reynolds, J.L. (1997). Post-traumatic stress disorder after childbirth: the phenomenon of traumatic birth. Canadian Medical Association Journal 156, 831-835.

[42] National Institute of Clinical Excellence (NICE). (2011). Guideline 132 issued in November 2011 (UK). Available at: http://guidance.nice.org.uk/CG132.

[43] Allport, G.W. (1964). Pattern and growth in personality. New York: Holt.

[44] Dweck, C.S. (1999). Self-theories: their role in motivation, personality and development. Philadelphia: Taylor and Francis/Psychology Press.

[45] Kaur, J. \& Kaur, K. (2012). Obstetric complications: Primiparity versus multiparity. European Journal of Experimental Biology 2 (5) 1462-1468.

[46] Fretts, R.C., Schmittdiel, M.A., McLean, F.H., Usher, R.H. \& Goldman, M.B. (1995). Increased maternal age and the risk of fetal death. New England Journal of Medicine 333, 953-957. 